\title{
CONTROL OF HVDC SYSTEMS OPERATING WITH LONG DC CABLES
}

\author{
Dragan Jovcic \\ University of Ulster, United Kingdom
}

\begin{abstract}
This paper studies dynamic aspects of HVDC system operation with long DC cables. The eigenvalue analysis of CIGRE HVDC system with extended DC cable is presented at the beginning. It is concluded that significant aggravation in the system stability is present with cables of $1000 \mathrm{~km}$ and the system dynamic instability is expected at frequencies $2 \mathrm{~Hz}<\boldsymbol{f}<4 \mathrm{~Hz}$. The design solutions based on smoothing reactors modifications or existing control loops re-tuning can not improve the system stability. Direct voltage feedback or AC current angle feedback at inverter side are the suitable control strategies. AC current angle is more favorable since the probability of commutation failure is reduced. The last section studies the operating conditions with low SCR inverter AC system and concurrently long DC cables. Eigenvalue analysis shows that this is particularly bad combination and resonance-like conditions rapidly aggravate system stability. Nevertheless, AC current angle feedback significantly improves the system stability. The analysis in the paper is accomplished using analytical system model and MATLAB software.
\end{abstract}

Keywords: HVDC control, HVDC modeling, Power system dynamic stability,

\section{INTRODUCTION}

Traditionally, HVDC transmission is employed mainly into three categories: medium length underwater cables (below $100 \mathrm{~km}$ ), long overhead transmission lines and connection of asynchronous systems (back to back schemes). The successful HVDC operation in these areas, over the last 5 decades, has signaled possible expansion of the HVDC application domain.

The recent restructuring of electrical energy market, and privatization of utilities, has brought forward many different concepts of power generation and different options for power transfers, including the transmission over long sea cables, Karlsson and Liss [1], Hammons et al [2]. HVDC appears as a viable candidate for long under sea transmission, however this technology is yet to be confirmed.

Reference [1] elaborates on a number of possible HVDC projects with sea cables of $500-700 \mathrm{~km}$, and points that some projects with $1000 \mathrm{~km}$ cables are being discussed.

It is expected that prolonged DC cables will alter DC system dynamic behavior and that stability of interconnected AC systems may also be affected. Reference [1] further summarizes that main control issues stem from the high cable capacitance and they are reflected in:

- Inability of rectifier controller to "see" beyond the high capacitance cable, i.e. inverter system end, leading to different rectifier and inverter responses,

- The discharge of huge cable energy that would lead to AC system collapse.

The above conclusions are derived using static $U d / I d$ HVDC indicators, and observed employing digital simulators, whereas they are not confirmed and studied using dynamic system analysis.

This paper discusses the HVDC operation with long DC cables, from the dynamic pint of view and primarily in the frequency domain $1 \mathrm{~Hz}<f<100 \mathrm{~Hz}$. The primary aim of this research is to offer eigenvalue-based analysis of the above phenomena. Using the eigenvalue decomposition analysis and studying the movement and sensitivity of oscillatory modes, it is possible to predict the frequency of possible instabilities and to derive qualitative conclusions about the stability margins.

The influence of the existing control strategies can also be studied by analysing the affect that changes in controller parameters have on dominant eigenvalues. In a similar way, the influence of other parameters like smoothing reactors size is investigated in order to advice best design options with long DC cables. A new HVDC control method that can counteract the observed stability problems will further be discussed. In the last section, the paper studies the dynamic implications of combining long $\mathrm{DC}$ cables and weak receiving $\mathrm{AC}$ systems.

The dynamic HVDC system model employed is presented by Jovcic et al [3]. The standard CIGRE HVDC model, as presented by Szechman et al [4] is used in the analysis as the starting test system and modified accordingly to represent long DC cable.

The study and results from this paper will assist the system designers and operators because of the generic type conclusions on expected dynamic difficulties and possible instabilities with long DC cables. They are of further importance since they signal directions for the development of future HVDC control strategies, particularly at inverter side. The results present preliminary theoretical confirmation of technical feasibility of long DC cable HVDC projects.

\section{TEST SYSTEM}


The starting test system used in the analysis section is the CIGRE monopolar HVDC system given in [4], and it is modeled as shown in [3], implemented in MATLAB software. The controller uses PI rectifier current controller and gamma controller at inverter side as given in PSCAD/EMTDC HVDC Benchmark model. To represent the operating changes with long DC cables, the following model parameters are varied:

- Cable capacitance $C=C_{c i g}+C_{i}$,

- $\quad$ DC line resistance $R_{d c}=R_{d c-c i g}+C_{i} * 10^{6} / 40,(2)$

where: $C_{i} \in\left[0,800 \times 10^{-6} \mu F\right], C_{c i g}, R_{d c-c i g}$-original

CIGRE value.

The above highest value for capacitance ( $C_{i}=800 \times 10^{-6} \mu F$ in (1)) is used in the system analysis as the expected capacitance for approximately $2000 \mathrm{~km}$ long cable. Although this length might not have practical significance, the study gives generic theoretical conclusions. In the controller design section, a more realistic value of $C_{i}=400 * 10^{-6} \mu F$ is used, representing approximately $1000 \mathrm{~km}$ cable and this system is termed original system in the design section.

DC line resistance is assumed to vary linearly with the variation in cable capacitance, as given by formula (2). It is noted that formula (1) and (2), derived using PSCAD simulation, are a crude approximation of the actual values, nevertheless the validity of conclusions is confirmed in sections III and IV.

In testing the results with PSCAD, a detailed frequency dependent cable model is used and only the cable length is varied.

\section{STABILITY ANALYSIS}

\section{III.1 Eigenvalue analysis of HVDC systems with long cables}

In this section, the cable capacitance and cable resistance are varied in small steps as given by (1) and (2), using the analytical system model and the location of eigenvalues is observed.

Figure 1 shows the root locus where crosses denote original CIGRE model eigenvalues. Observing the movement of eigenvalues at higher frequencies (branches $a$ and $b$ ) it is seen that long cables do not significantly alter higher frequency stability. These branches are short and stability is somewhat improved. In mid frequency range (branch $c$ ) the frequency of the original mode is altered and the system stability is somewhat degraded. Finally, at lower frequencies, a significant degradation in the system stability occurs, as seen by the root branch $d$.

The end value of eigenvalue branch $d$ (location A1) is dynamically unstable, confirming that system becomes unstable with cable length around $1500 \mathrm{~km}$. The eigenvalues move slower as the cable length is increased, and the eigenvalue location for approximately $1000 \mathrm{~km}$ long cable (location A2) is very close to the instability.

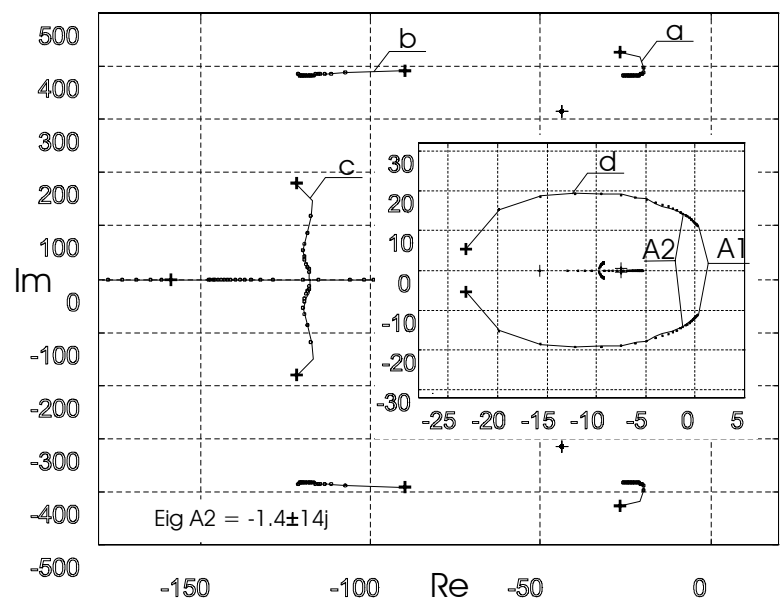

Figure 1. Root locus with the increase in DC cable capacitance and resistance. “+”- Standard CIGRE model.

The system responses are expected to be very poor with $200-300 \mathrm{~km}$ long cables since eigenvalues move very fast for lower cable lengths.

The frequency for possible instabilities is $2 \mathrm{~Hz}<f<4 \mathrm{~Hz}$. It should be noted that long DC cables do not significantly aggravate damping of oscillatory mode close to first harmonic $(5 \mathrm{OHz})$, and therefore composite resonance and second harmonic instability is not accelerated.

The above conclusions are confirmed with PSCAD/EMTDC simulation, as shown in Figure 2. A single dominant oscillatory mode is evident and it also seen that inverter side is much more prone to the oscillations. The figure further verifies the analytical model, pointing also that model can not perfectly match non-linear digital simulation.

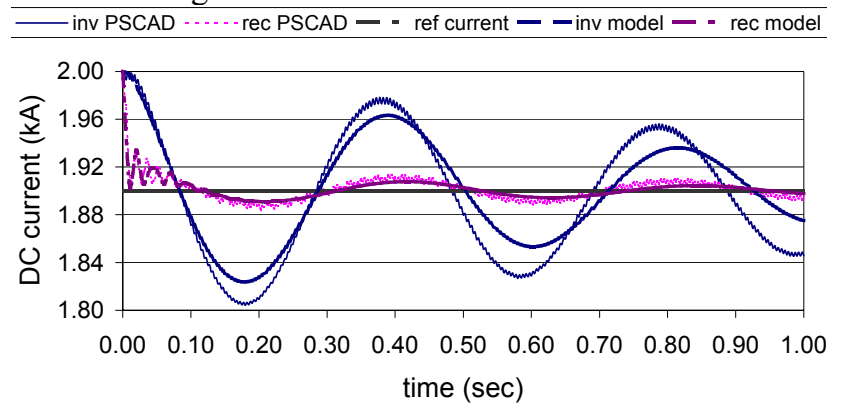

Figure 2. $1000 \mathrm{~km}$ cable HVDC system, DC current (rectifier and inverter) response following a current reference step change.

\section{III.2 Influence of Existing Control Loops}

The existing rectifier controller gains (proportional and integral) are varied in order to improve dynamics of the system with long DC cables. The method of analysis follows the classical root locus method for HVDC systems as described by Jovcic et al [5]. The results are similar to those obtained for rectifier DC current 
feedback in this reference. In summary, with the existing HVDC control structure, it is not possible to improve the system stability without destabilizing other modes or reducing the speed of the response.

\section{III.3 Influence of Smoothing Reactors}

Smoothing reactors are normally added to attenuate DC harmonics and to limit the rate of DC current responses in cases of DC faults and DC side overvoltages. In line with the same design method, the value of smoothing reactors is further increased in order to compensate for large cable capacitance and to attenuate DC current responses.

The value of inductance of smoothing reactors is varied in the following manner:

$$
\begin{aligned}
& L_{d c}=L_{d c-c i g}+L_{i}, \\
& L_{i} \in[0,1 H], L_{d c-c i g} \text {-original CIGRE value. }
\end{aligned}
$$

Figure 3 shows the root locus, where the final eigenvalue locations (A1, B1, C1 and D1) correspond to $L_{i}=1 H$. The original system corresponds to $C_{i}=400 * 10^{-6} \mu F$ (A2 location in Figure 1).

It is see that increase in smoothing rectors significantly slows system responses, as demonstrated by reduced frequency in $c$ branch. However the damping of the dominant eigenvalues is also reduced and the system is further destabilised. Is is evident that soleley increase in smoothing recators can not improve dynamic stability of the systems with long DC cables. Reducing the smothing reactors marginally enhabces the stability.

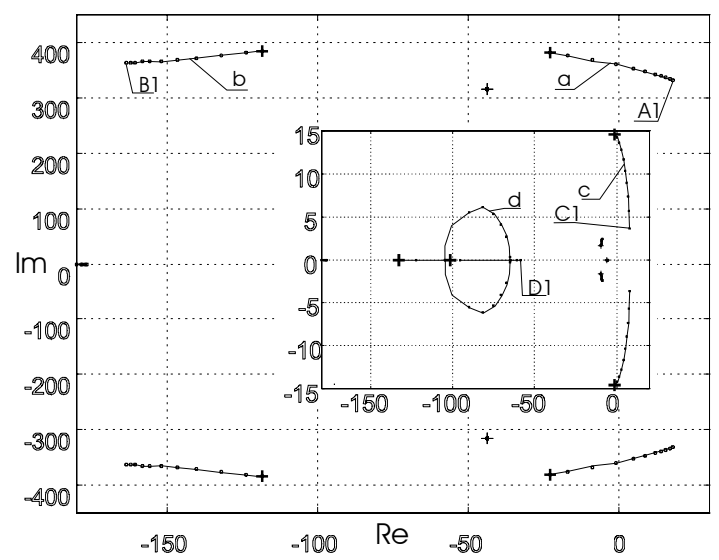

Figure 3. Root locus with the increase in DC smoothing reactance. "+" - Original system with long DC cable.

\section{CONTROLLER DESIGN}

\section{IV.1 Design based on stability requirements}

In this section, various inverter control strategies are examined in an attempt to improve the system stability. The original system corresponds to A2 eigenvalue location in Figure 1. The main design goal is to improve damping of eigenvalues A2 without stability deterioration at other frequencies (other modes). The method of analysis resembles the one used in [5].

Most inverter variables are studied as feedback candidate signals including: $\mathrm{AC}$ voltage, $\mathrm{AC}$ current angle, DC voltage, DC current. Only two feedback strategies are found to improve the system stability: Direct voltage feedback (DCV) and AC current angle (ACCA) feedback, and they are further examined and compared. It is noted that AC current angle feedback is earlier found to be effective inverter control strategy with weak receiving $\mathrm{AC}$ systems, as presented by Jovcic et al [6].

Figures 4 and 5 show the root locus with DCV and ACCA feedback respectively. It is seen that both control strategies improve damping at lower frequencies (branch $c$ in Figure 4 and $a$ in Figure 5) and system stability is not jeopardized at higher frequencies. More precisely, the negative influence at higher frequencies with DCV feedback is evident by observing branch $a$ in Figure 4, however the system is still stable with high gain values and this negative effect can be compensated with a feedback filter. DVC will have faster responses since branch c moves towards higher frequencies. Therefore, both control strategies can be used for system operation with long DC cables.
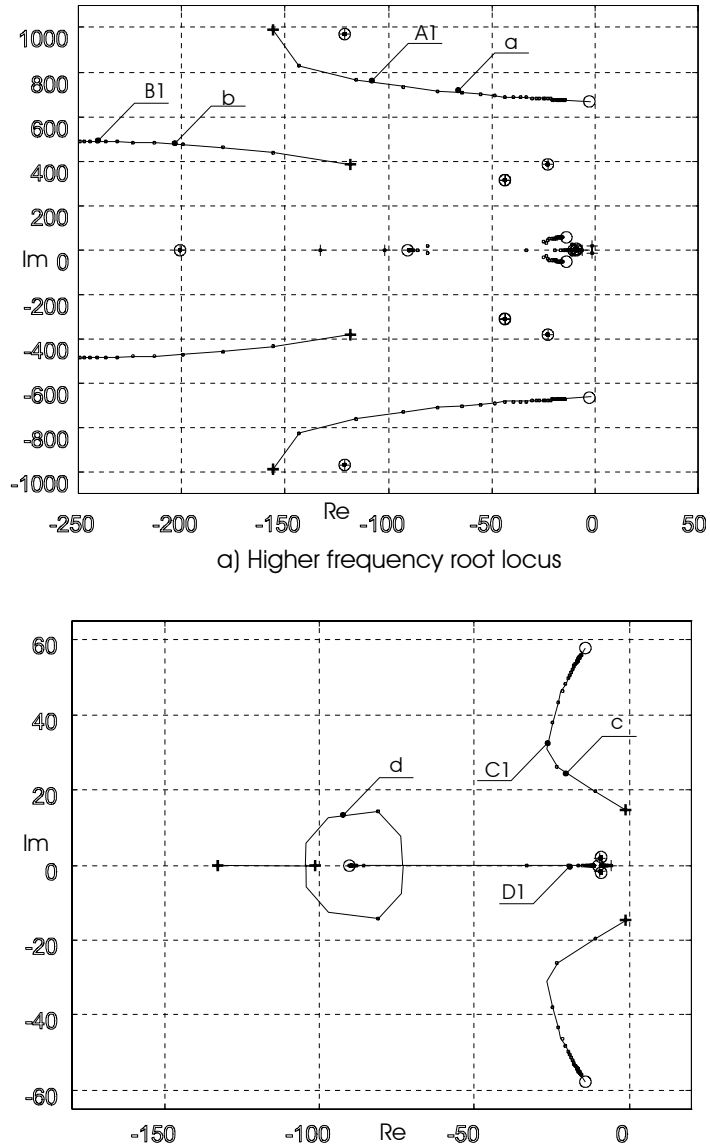

b) Lower frequency root locus

Figure 4. Root locus with direct voltage feedback. "+” - Original eigenvalues with long DC cable. 


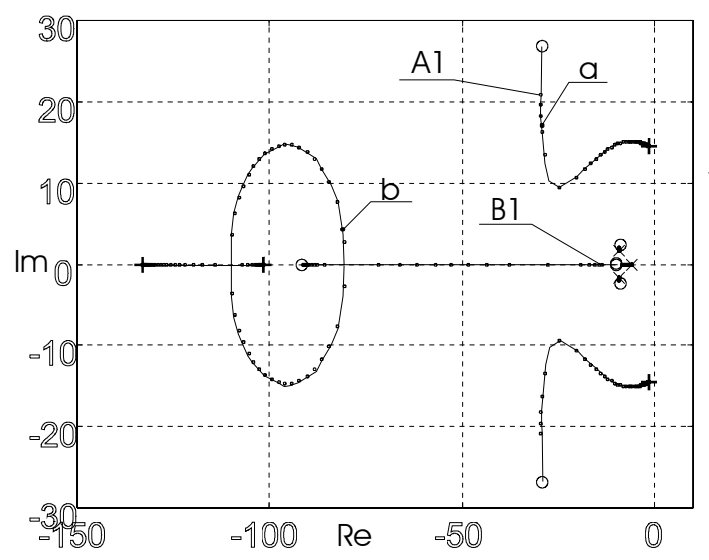

Figure 5. Root locus with AC current angle feedback. “+”- Original eigenvalues.

Figure 6 compares the time domain responses for the two feedback loops. The exact value of the feedback gain is selected such that the eigenvalue location corresponds to the position marked A1, B1, C1 and D1 in Figure 4 and A1, B1 in Figure 4. Using the classical control theory recommendations, DCV feedback would be selected from Figure 6 since the responses are somewhat faster and the overshoot is within $20-25 \%$ boundary.

However to fully reflect the particular requirements of the HVDC system, especially commutation failure considerations, the disturbance responses for $\mathrm{AC}$ and DC variables are studied as shown in the next section.

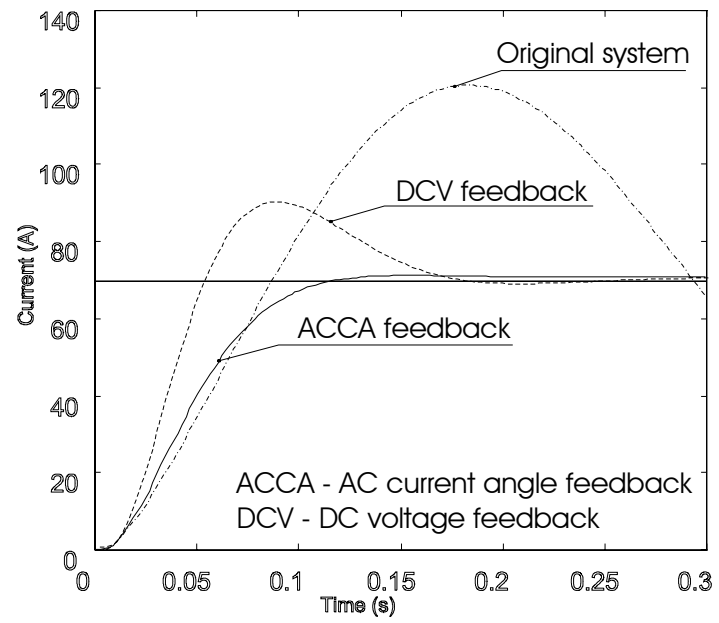

Figure 6. Inverter DC current response after current reference step change. Rectifier DC current responses are not influenced with feedback.

\section{IV.2 Commutation failure considerations}

This section compares the DCV and ACCA feedback by considering the probability of commutation failure.

It is known that commutation failure is caused by reduction in gamma values, which is primarily caused by $\mathrm{AC}$ voltage depression and $\mathrm{DC}$ current increase at inverter side, as shown by Thio et al [7].

Figure 7 compares the inverter DC current and $\mathrm{AC}$ voltage responses for the two feedback loops and considering the disturbance at inverter AC system (tap changer action). It is seen that in the interval 0.02$0.04 \mathrm{sec} \mathrm{DC}$ current values are higher and $\mathrm{AC}$ voltage values are lower for DCV feedback. This result leads to conclusion that probability of commutation failure is higher with DCV feedback than with ACCA feedback and consequently ACCA feedback loop would be more favorable.

It is therefore concluded that although both control strategies improve the system stability, considering the commutation failure probability, ACCA feedback is better option.

It should be noted that both feedback control strategies in the above analysis use simple feedback gain without any feedback filter. This is contrary to the ACCA control employed in [5], which uses Hinf controller in ACCA feedback loop to maximise the feedback control effect.

Figure 8 shows the PSCAD simulation of the above control method. Figure 8 a) shows that the ACCA feedback control gives quite acceptable responses $(25 \%$ overshoot and $0.2 \mathrm{~s}$ settling time) with $100 \mathrm{~km}$ DC cable. Figure $8 \mathrm{~b}$ ) confirms that $\mathrm{AC}$ current angle stabilises the $2000 \mathrm{~km}$ system, the responses are somewhat sluggish though (settling time of $0.5 \mathrm{~s}$ ).

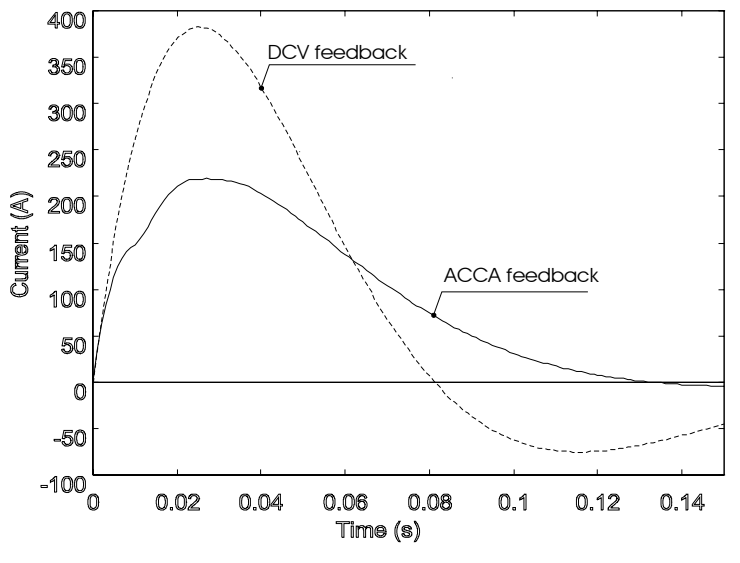

a) Inverter DC cursent response

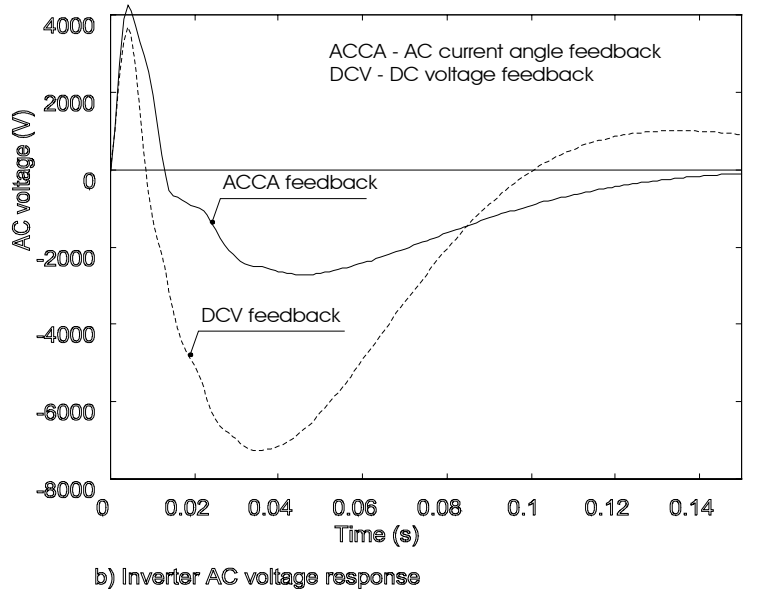

Figure 7. System response following disturbance on inverter $A C$ voltage 


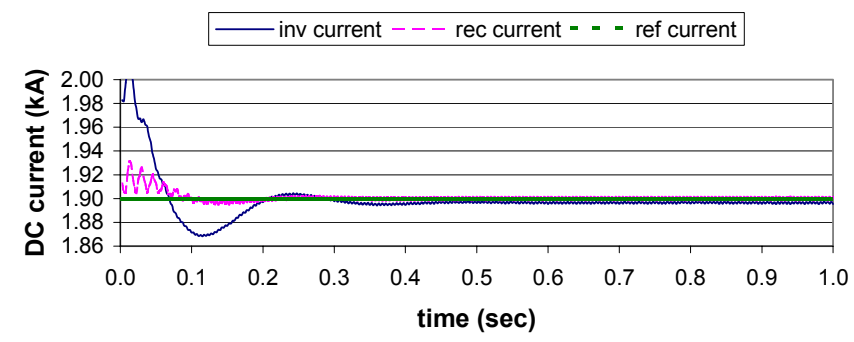

a) $1000 \mathrm{~km} \mathrm{DC} \mathrm{cable}$

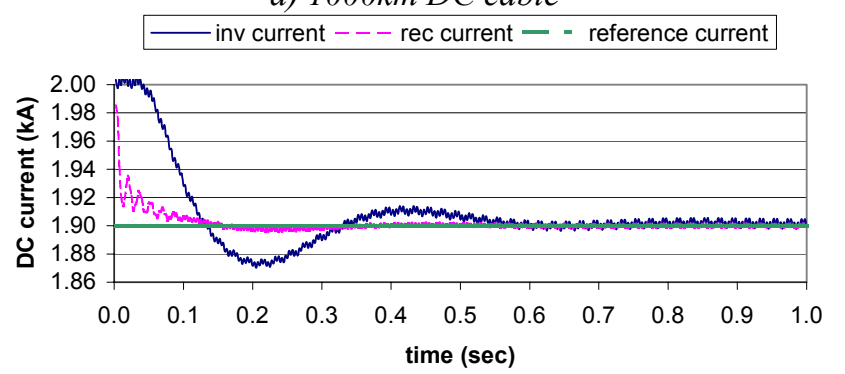

b) $2000 \mathrm{~km} \mathrm{DC} \mathrm{cable.}$

Figure 8. PSCAD simulation of long cable HVDC system with supplementary inverter control. System response following a step change in reference current.

The simulation with PSCAD confirms the above generic conclusions, nevertheless because of the presence of noise and non-linear elements, the actual value of feedback controller gains is restricted. As a result, a slightly slower responses and larger overshoots, compared with responses with analytical model in Figures 6 and 7, are obtained.

\section{VERY WEAK RECEIVING AC SYSTEMS AND LONG DC CABLES}

\section{V.1 System analysis}

This section studies the case of long DC cables and simultaneously very weak receiving AC system. Using the static analysis and simulation, this operating condition is shown to be very difficult [1], however this reference does not offer dynamic system analysis to support the conclusion.

Reference [6] concludes that weak AC systems will cause dynamic instabilities in the frequency range $2 \mathrm{~Hz}<f<7 \mathrm{~Hz}$. Since this frequency range is close to the dynamic instability domain with long DC cables, as demonstrated by branch $d$ in Figure 1, the concern is now raised that combination: long DC cables with weak AC systems will cause rapid worsening in the system stability.

Table 1 shows the dominant eigenvalues for very weak receiving AC system, and $600 \mathrm{~km}$ long DC cable case. In the first column, only the inverter AC system SCR is reduced to $S C R=1.3$, and the dominant oscillatory mode at frequency around $3 \mathrm{~Hz}$ is evident. In the second column, only the DC cable is extended (with original $S C R=2.5$ ) to approximately $600 \mathrm{~km}$, creating a complex eigenvalue pair with frequency very close to $3 \mathrm{~Hz}$, i.e. close to earlier case with low SCR. Therefore, both operating conditions degrade system stability in the same frequency range, although the system is stable in both configurations. The last column in the Table confirms that the system becomes unstable if the system configuration with $S C R=1.3$ and $600 \mathrm{~km}$ long DC cable is used.

It can be concluded that low inverter SCR and long DC cable is particularly difficult combination for system dynamic stability. This operating conditions can lead to specific "resonance" at frequencies around $3 \mathrm{~Hz}$. The above mechanism for instability is very similar to the known HVDC composite resonance as shown by Wood and Arrillaga [8]. A specific condition on AC side (low SCR) combined with a corresponding condition on DC side (long DC cable) will lead to dynamic instabilities in particular frequency range.

\section{V.2 AC current angle feedback}

Figure 9 shows the use of ACCA feedback with the system having weak AC system and long DC cable. The original system is the configuration from third column, Table 1. It is seen that ACCA can stabilise the system and the step responses are satisfactory.

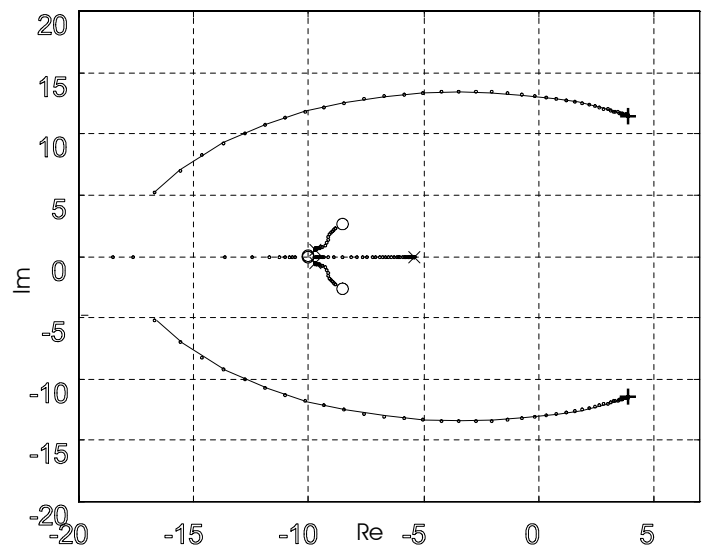

a) Root locus

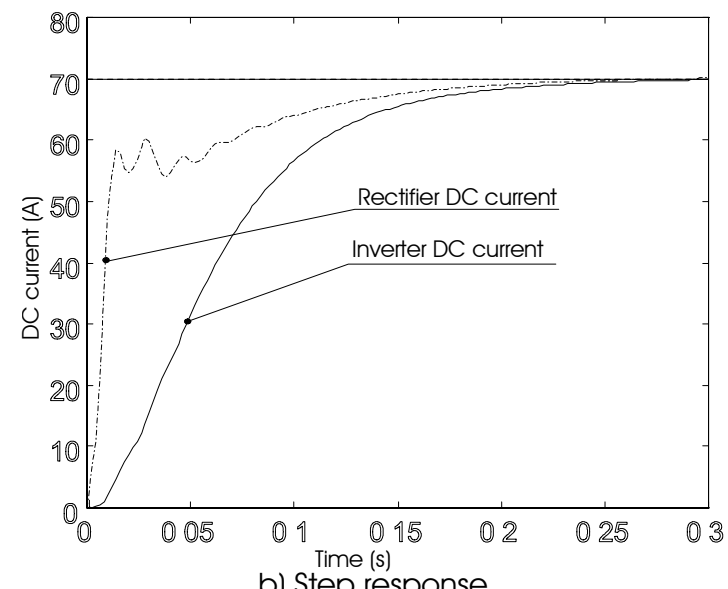

b) Step response

Figure 9. System with low SCR and long DC cables. Root locus and step response with ACCA feedback. "+" Original system. 
TABLE 1 - DOMINANT EIGENVALUES FOR LOW SCR AND LONG DC CABLE

\begin{tabular}{|l|l|l|l|}
\hline $\begin{array}{l}\text { System } \\
\text { Configur. }\end{array}$ & $\begin{array}{l}\text { Inverter AC } \\
S C R=1.3\end{array}$ & $\begin{array}{l}S C R=2.5, \\
600 \mathrm{~km} \text { DC cable }\end{array}$ & $\begin{array}{l}S C R=1.3, \\
600 \mathrm{~km} \text { DC cable }\end{array}$ \\
\hline $\begin{array}{l}\text { Dominant } \\
\text { eigenvalue }\end{array}$ & $-5.1 \pm 17.27 \mathrm{j}$ & $-5.3 \pm 17.98 \mathrm{j}$ & $+3.8 \pm 11.50 \mathrm{j}$ \\
\hline
\end{tabular}

It is further noted that in Figure 9 original proportional gain at rectifier side is reduced by half in order to improve damping of oscillatory mode close to first harmonic.

It should be observed in Figure 9 b) (also in Figure 8) that inverter DC current response is noticeably lagging the rectifier current response. This is a consequence of high DC cable capacitance that contributes decoupling rectifier from inverter. For this reason, solely used rectifier controller is not effective for long cable configurations.

\section{CONCLUSIONS}

Eigenvalue analysis of HVDC system operating with long DC cables shows that significant aggravation in the system stability occurs with cables of around $1000 \mathrm{~km}$. The system dynamic instability may occur at frequencies $2 \mathrm{~Hz}<f<4 \mathrm{~Hz}$. The analysis further shows that design solutions based on smoothing reactors modifications or existing control loops re-tuning can not improve the system stability. Direct voltage feedback and $\mathrm{AC}$ current angle feedbacks are suitable control strategies. The study of commutation failure probability verifies that $\mathrm{AC}$ current angle is more favorable since the DC current and $\mathrm{AC}$ voltage overshoots are reduced. PSCAD simulation in general confirms that AC current angle feedback can give satisfactory responses with $1000 \mathrm{~km}$ DC cable. The last section studies the operating conditions with low SCR inverter AC system and simultaneously long DC cables. Eigenvalue analysis shows that this is particularly bad combination and resonance-like conditions rapidly deteriorate the system stability. Nevertheless, AC current angle feedback at inverter side significantly improves the system stability. This feedback strategy will move the unstable eigenvalues into stable region and it will not influence other eigenvalues.

\section{REFERENCES:}

[1] T.Karlsson, G.Liss, 1995, "HVDC Transmission with extremely long DC cables control strategies" IEEE/KTH Stockholm Power Tech Conference, Stockholm pp 24-29.

[2] Hammons, K.O.Lee, K.H. Chew and T.C. Chua, 1998, "Competitiveness of renewable energy from Iceland via the proposed Iceland/UK HVDC submarine cable link". Electric Machines \& Power Systems, vol.26, no.9, pp.917-33.
[3] D.Jovcic N.Pahalawaththa, M.Zavahir, April 1999 "Analytical Modeling of HVDC Systems" IEEE Transactions on PD, Vol. 14, no 2, pp. 506-511.

[4] M. Szechman, T. Wess and C.V. Thio, April 1991, "First Benchmark model for HVDC control studies", CIGRE WG 14.02 Electra No. 135, pages: 54-73.

[5] D.Jovcic N.Pahalawaththa, M.Zavahir, March 1999, "Stability Analysis of HVDC Control Loops" IEE Proceedings Generation, Transmission and Distribution, Vol. 146, no 2, pp. 143-148.

[6] D.Jovcic N.Pahalawaththa, M.Zavahir, May 1999, "Inverter Controller for Very Weak Receiving AC Systems" IEE Proceedings - Generation, Transmission and Distribution, Vol. 146, no 3, pp. $235-240$

[7] C.V. Thio, J.B. Davis, K.L. Kent, 1995, "Commutation Failures in HVDC Transmission Systems" Paper presented at IEEE/PES Summer meeting 1995. 95 SM 377-2 PWRD.

[8] A.R. Wood Arrillaga, Oct 1995, Composite resonance; a circuit approach to the waveform distortion dynamics of an HVDC converter, IEEE PWRD, vol 10, no 4, pp 1882 to 1888. 